\title{
Changes of size and shape of small, unruptured intracranial aneurysms in repeated computed tomography angiography studies
}

\author{
Jarosław Żyłkowski ${ }^{1}$, Przemysław Kunert ${ }^{2}$, Maciej Jaworski ${ }^{1}$, Grzegorz Rosiak ${ }^{1}$, Andrzej Marchel ${ }^{2}$, Olgierd Rowiński ${ }^{1}$ \\ ${ }^{1}$ Second Department of Radiology, Medical University of Warsaw, Warsaw, Poland \\ ${ }^{2}$ Chair and Department of Neurosurgery, Medical University of Warsaw, Warsaw, Poland
}

Videosurgery Miniinv 2015; 10 (2): 178-188

DOI: $10.5114 /$ wiitm.2015.52707

\begin{abstract}
Introduction: Unruptured intracranial aneurysms (UIAs) are frequently detected in noninvasive imaging studies such as computed tomography angiography (CTA) or magnetic resonance angiography (MRA). If small, UIAs are observed in these modalities in order to detect growth or shape change, but there are many questions about proper protocol of the follow-up.

Aim: To assess changes of small $(<7 \mathrm{~mm})$ UIAs dome size and shape in repeated CTA studies as predictors of growth and rupture.

Material and methods: One hundred and ten UIAs (10 posterior circulation) in 70 patients (55 women) were observed, with a cumulative observation time of 333.32 years. Aneurysms' dome and neck perpendicular dimensions were measured in the first and the last CTA study at least twice with the developed application. Confidence intervals (CI) for measurements and dome shape parameters were calculated. For aneurysms ruptured during follow-up intermediate studies were analyzed. Patients' clinical information was recorded. The aneurysm growth detection algorithm integrated $\mathrm{Cl}$ and spatial resolution of the CT scanner.

Results: Twenty-three aneurysms increased in volume, 10 in height and 14 in dome width. Volume increased in 90\% of cases of height and $93 \%$ of width increase. Posterior circulation aneurysms grew faster than anterior ones ( $p<0.003)$, but calculated time to significant size increase ( $\mathrm{eT})$ did not differ between the groups due to higher $\mathrm{Cl}$ in the posterior circulation. Analysis of ET with Kaplan-Meier curves showed that $75 \%$ of growing aneurysms could be detected in the first 3 years of observation. During the follow-up 3 aneurysms bled, and they grew faster than other growing aneurysms. Two of the bleeding aneurysms formed daughter sacs.

Conclusions: Dome volume assessment is superior to single dimension assessment in aneurysm growth detection. Confidence intervals assessment helps to avoid overestimation of growth. Seventy-five percent of growing aneurysms could be detected in the first 3 years of observation. Daughter sac formation and fast increase in size are strong predictors of aneurysm rupture.
\end{abstract}

Key words: unruptured intracranial aneurysm, computed tomography angiography, dome size, dome shape.

\section{Introduction}

Risk of bleeding from intracranial aneurysms varies depending on their initial size, location, positive history of subarachnoid hemorrhage (SAH) and irregularity of the sack [1-3]. The lowest risk was found for small, regular aneurysms located in the anterior circulation, especially in the cavernous segment of the internal carotid artery (ICA) and bifurcation of the middle cerebral artery (MCA) [2, 3].

Rupture of intracranial aneurysm causes SAH, presenting high morbidity and fatality [4, 5]. Preventive treatment is possible but the risk-benefit for 
each case should be weighed [6-9]. For very small aneurysms, even in the posterior circulation, in most cases this factor does not justify any type of treatment. These patients are followed with non-invasive imaging techniques such as computed tomography angiography (CTA) or magnetic resonance angiography (MRA). The aim of this strategy is to detect an aneurysm growth, which was identified by researchers as a sign of dome instability and a factor increasing risk of rupture [2, 10-12].

Although this type of observation is widely practiced, there are many important questions about it. The optimal interval between studies is not estimated. There are many strategies of measurement and detection of aneurysm growth [13-18]. Other than initial aneurysm volume $[13,19,20]$ and daughter sac formation $[13,21,22]$ risk factors for aneurysm growth and rupture are not defined or confirmed. Effectiveness of this method in preventing $\mathrm{SAH}$ is unknown.

In this study we present our experience, strategy, improvements of measurement methods and conclusions from almost 7 years of observation of small, unruptured aneurysms in our institution.

\section{Aim}

The aim of the study was to assess changes of small (less than $7 \mathrm{~mm}$ in maximal diameter), unruptured aneurysms' dome shape and size during follow-up with CTA studies as predictors of their growth and rupture.

\section{Material and methods}

\section{Methods}

Patients of the Department of Neurosurgery being observed because of small, unruptured intracranial aneurysms were reviewed. Individuals who have had done at least two, good quality CTA studies in interval of at least 100 days were selected. Available studies were retrieved from Department of Radiology PACS system and stored in international DICOM format on local server for analysis.

Measurements were performed using dedicated application designed and written by one of our team members. The application presented three-dimensional (3D) data in four, independent viewports as reformatted images. In case of difficult anatomy, there was also a possibility to present anatomical structures as 3D models utilizing marching cubes algorithm. Two dedicated measuring tools were implemented: one for dome and one for neck measurements. Both tools allow for strictly perpendicular measurements of 3 dimensions of the dome and 2 of the neck.

Height of the aneurysm was posited as a line perpendicular to vessel axis at the level of aneurysm neck. In case of highly inclined and elongated dome we posited height as the longest line connecting center of neck with the dome surface.

The neck was measured in a plane tangential to the parent vessel at the level of aneurysm neck. For aneurysms with shape close to hemisphere this plane was placed as close to vessel wall as possible. For aneurysms of ellipsoid-like shape the plane was placed in the narrowest section of the base of the dome.

Each aneurysm was assessed in the first and last available study at least twice by one observer. For each round of measurements each aneurysm was measured in the first and last study in one session. The interval between each round of measurements was about 4 weeks. Collected data were semiautomatically linked with the patient, aneurysm and study in the database. After linkage data were automatically stored in the database (PostgreSQL 9.2 engine).

For aneurysms which ruptured during the period of observation, additional measurements were performed for all available studies in order to perform a detailed analysis of their size changes in time.

Calculations of the volume of the aneurysms were based on the equation of spheroid volume (equation 1).

Average dome diameter $(W)$ and average neck diameter $(N)$ were calculated as the geometric mean of two perpendicular measurements (equation 2).

Calculation of average volume, height, dome and neck diameter and also standard deviation for each measured dimension for each study were done by database logics.

For each selected patient medical data concerning hypertension, smoking, autosomal dominant polycystic kidney disease (ADPKD), family history of aneurysms, previous SAH and other aneurysms' treatment were collected and stored in the database.

Data from the database were extracted in three separate SQL queries first for all aneurysms, second for patients' medical and demographic data 


$$
V=\frac{4}{3} \pi \cdot \frac{H}{2} \cdot \frac{W_{1}}{2} \cdot \frac{W_{2}}{2}
$$

after reduction:

$$
V=\frac{\pi \cdot H \cdot W_{1} \cdot W_{2}}{6}
$$

Equation 1. Equation of aneurysm dome volume calculation: $H$ - height of the dome, $W_{1}$ and $W_{2}$ perpendicular, transverse dimensions of the dome.

$$
g a A=\sqrt{A_{1} \cdot A_{2}}
$$

Equation 2. Geometric average calculation: gaA - geometric average of $A_{1}$ and $A_{2}$.

$$
\begin{aligned}
& \operatorname{sign} \Delta H=\max \left(C I_{H} ; 0.625 \mathrm{~mm}\right) \\
& \operatorname{sign} \Delta W=\max \left(C I_{W} ; 0.625 \mathrm{~mm}\right)
\end{aligned}
$$

Equations 3. Calculations of significant $H(a)$ and $W(b)$ change.

$$
\begin{aligned}
& \operatorname{scV}_{H}=\frac{\pi \cdot(H+\operatorname{sign} \Delta H) \cdot W^{2}}{6} \\
& \operatorname{scV} V_{H}=\frac{\pi \cdot H \cdot(W+\operatorname{sign} \Delta H) \cdot W}{6}
\end{aligned}
$$

Equations 4. Equations of aneurysm volume after significant change of $H(a)$ and $W(b)$ dimensions.

$$
\operatorname{maxcV}=\max \left(s c V_{H} ; s c V_{w}\right)
$$

Equation 5. Algorithm for selection of maximal aneurysm dome volume after significant change of one of the dimensions (maxcl).

$$
\min \Delta V=\left|V_{1}-\operatorname{maxc} V\right|
$$

Equation 6. Calculation of minimal, absolute $\Delta V$ $(\min \Delta V) . V_{1}$ - aneurysm volume from the first CTA study.

$$
\operatorname{sign} \Delta H=\operatorname{MAX}\left(\min \Delta V ; C l_{V}\right)
$$

Equation 7. Significant $\Delta \mathrm{V}$ calculation.

$$
e T_{x}=\frac{C I_{x}}{\frac{d X}{d T}}
$$

Equation 8. $e T_{X}$ - expected time to significant change of value of analyzed parameter (X), $\mathrm{Cl}_{x}$ - confidence interval for particular measurements, $\mathrm{d} X / \mathrm{d} T$ - calculated speed of analyzed value change. and third for aneurysms ruptured during follow-up. Further calculations were done in MS Excel (Microsoft). For each aneurysm geometric parameters such as aspect ratio $(A R)$, bottle neck ratio $(B N)$ and height to average width ratio (HW) (Figure 1) and also change of volume $(\mathrm{d} V)$, height $(\mathrm{d} H)$ and average dome width ( $\mathrm{d} W$ ) between the first and last study were calculated as well as the speed of their change (respectively: $\mathrm{d} V / \mathrm{d} T, \mathrm{~d} H / \mathrm{d} T$ and $\mathrm{d} W / \mathrm{d} T$ ) as a base unit per day. The relative parameter of percentage of initial volume change (\%dV) and its speed of change per day was calculated $(\% \mathrm{~d} V / \mathrm{d} T)$.

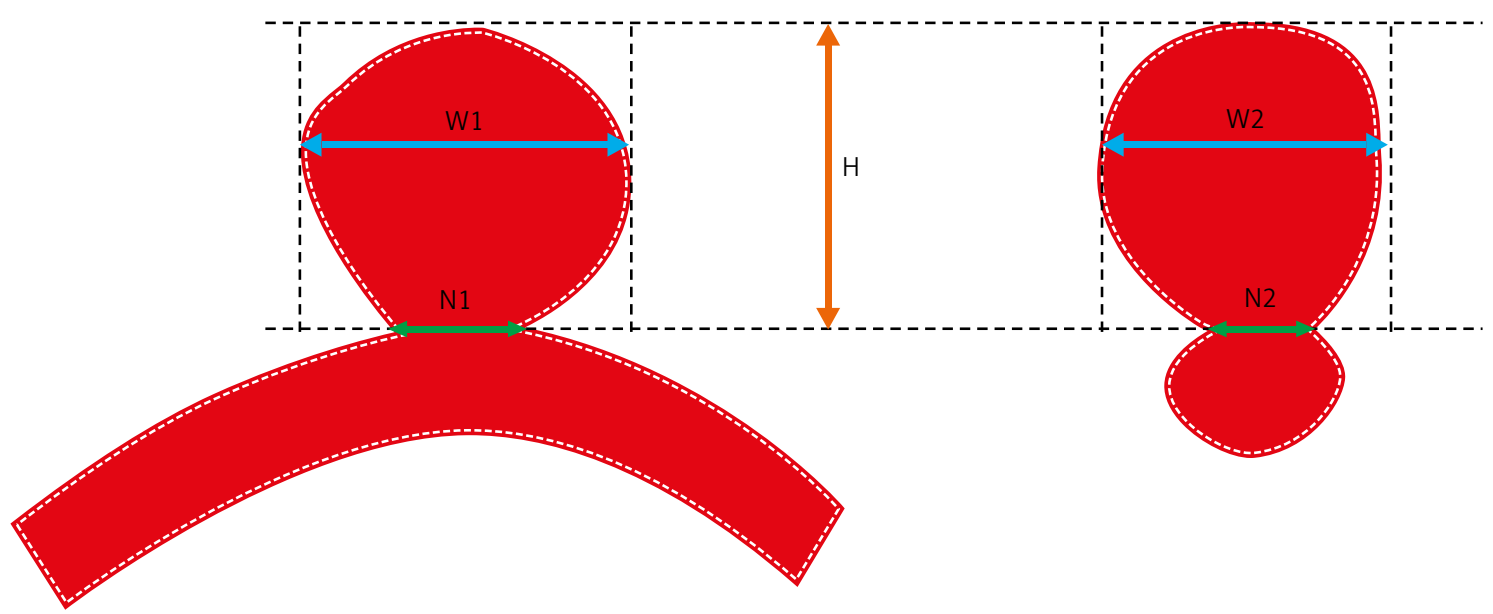

Figure 1. Two perpendicular views of the dome of the aneurysm

Explanation of dome size and shape parameters: $H$-dome height, $W$-dome width, $N$-neck width, $A R=H / N, B N=W / N$ and $H W=H / W$. 
The aneurysms were divided into groups depending on the change of dimensions (separately for each $\mathrm{d} V, \mathrm{~d} H$ and $\mathrm{d} W$ ). We assumed that $H$ and $W$ values change significantly in observation if the absolute difference between the first and the last study is greater than the sum of confidence intervals (Cls) with $\alpha=0.99$ for measurements for each study but not less than the voxel size of the CT scanner, which was $0.625 \mathrm{~mm}(\operatorname{sign} \Delta H$ and $\operatorname{sign} \Delta W$, equation 3$)$.

For $V$ analysis the sum of Cls from the first and last study $\left(\mathrm{Cl}_{V}\right)$, with $\alpha=0.99$, was calculated. After that, relying on calculated $\mathrm{Cls}$ for $H$ and $W$, aneurysm volume for significant change of $H\left(\mathrm{scV}_{H}\right)$ and $W\left(s c V_{w}\right)$ were calculated using equations $4 a$ and $4 b$.

The maximal value of $s c V$ was selected (equation 5) and minimal, absolute $\Delta \mathrm{V}$ was calculated $(\min \Delta V$, equation 6).

Significant $\Delta V$ was calculated as the maximum of $\min \Delta V$ and $C I_{V}$ (equation 7).

As for $H$ and $W$ analysis, if the condition of significant value change was not met, we assumed that values did not differ significantly and that the analyzed parameter was stable over the period of observation.

\section{Statistical analysis}

The final step of the statistical analysis was performed in Statistica version 10 (StatSoft Inc., Tulsa, OK, USA). Before each step of statistical analysis requiring normal distribution of analyzed parameters, this distribution was tested with the Lilliefors test. The null hypothesis that the distribution is not normal was excluded in all cases with 0.95 significance.

\section{Material}

Computed tomography angiography studies were performed between August 2006 and December 2013 on a GE LightSpeed 16 CT scanner in the Department of Radiology. Contrast medium (iomeprol-400) was administered intravenously in a volume of $60 \mathrm{ml}$ with injection speed of $6 \mathrm{ml} / \mathrm{s}$ with a pre- and post-injection bolus of $0.9 \%$ saline solution (10 $\mathrm{ml}$ and $20 \mathrm{ml}$ respectively) at the same speed of infusion. Scanning start was triggered automatically with the region of interest (ROI) placed in the ascending aorta.

Seventy patients (55 females) aged from 36.2 to 79.2 years, average 57.83 years (male: average 57.55 years, female: average 58.85 years) were in- cluded in the study. Twenty-two (31.4\%) of the observed patients had previously SAH from another aneurysm. In this group 18 were female. All bleeding aneurysms were treated. Additionally, 20 patients underwent elective treatment of other aneurysm. In the previously treated group 32 individuals underwent neurosurgical treatment, 6 endovascular and 4 using both techniques.

Twenty (28.6\%) patients had single aneurysms. The percentage of multiple aneurysms was higher in men than in women (80.0\% vs. $69.1 \%)$ but the difference was not statistically significant $\left(\chi^{2}=0.4071\right)$.

Generally accepted risk factors for aneurysm rupture in the analyzed group are presented in Table I.

One hundred and ten aneurysms were observed. Minimal time of the observation was 112 days, and maximal was 2429 days with an average of 1090.9 days. Average observation time was longer in women than in men (1148 days vs. 973 days). Cumulated time of observation was 333.32 aneurysm-years.

Distribution of observed aneurysms is presented in Table II.

\section{Results}

\section{General characteristics of observed aneurysms}

Results of the geometric analysis of observed aneurysms are presented in Table III. The aneurysms were mainly small, hemisphere-like aneurysms, less than $7 \mathrm{~mm}$ in the maximal dimension.

No statistically significant differences in size and shape between the male and female group $(p \geq 0.418)$ and anterior and posterior circulation aneurysms $(p>0.138)$ were found in $t$-tests.

Comparison of dome size parameters for distinct locations of aneurysms reveals that dome and neck width were highest for BA tip aneurysms. BA tip, ICA cave (C5), ICA ophthalmic (C6) and ICA posterior com-

Table I. Risk factors

\begin{tabular}{|lccc|}
\hline Risk factor & $\begin{array}{c}\text { Both } \\
\text { sexes }\end{array}$ & Female & $\begin{array}{c}\text { Percentage } \\
\text { of total }\end{array}$ \\
\hline Hypertension & 16 & 13 & 22.86 \\
\hline Smoking & 10 & 8 & 14.29 \\
\hline ADPKD & 5 & 5 & 7.14 \\
\hline $\begin{array}{l}\text { Familial history } \\
\text { of SAH }\end{array}$ & 1 & 1 & 1.43 \\
\hline
\end{tabular}

ADPKD - Autosomal dominant polycystic kidney disease. 
Table II. Aneurysms' localization and distribution in men and women groups

\begin{tabular}{|lccccccc|}
\hline Artery & \multicolumn{2}{c}{ Total } & \multicolumn{2}{c}{ Women } & \multicolumn{2}{c|}{ Men } \\
\cline { 2 - 8 } & $n$ & $\%$ & $n$ & $\%$ & $n$ & $\%$ \\
\hline ICA & 45 & 40.91 & 35 & 39.77 & 10 & 45.45 \\
\hline MCA & 34 & 30.91 & 28 & 31.82 & 6 & 27.27 \\
\hline ACoA & 16 & 14.55 & 13 & 14.77 & 3 & 13.64 \\
\hline BA quadrifurcation & 7 & 6.36 & 4 & 4.55 & 3 & 13.64 \\
\hline ACA & 5 & 4.55 & 2 & 2.27 & 0 & 0 \\
\hline PCA & 2 & 1.82 & 2 & 2.27 & 0 & 0 \\
\hline PCoA & 1 & 0.91 & 1 & 1.14 & 0 & 0 \\
\hline
\end{tabular}

ICA - Internal carotid artery, MCA - medial cerebral artery, ACOA - anterior communicating artery, BA - basilar artery, ACA - anterior cerebral artery, $P C A$ - posterior cerebral artery, $P C O A$ - posterior communicating artery.

Table III. Geometric characteristics of analyzed aneurysms

\begin{tabular}{|lccccc|}
\hline Variable & Average & Median & Minimum & Maximum & Std. dev. \\
\hline V & 25.60 & 17.07 & 2.39 & 133.69 & 26.54 \\
\hline$H$ & 2.79 & 2.54 & 0.77 & 6.30 & 1.14 \\
\hline$W$ & 3.70 & 3.58 & 1.81 & 6.86 & 1.11 \\
\hline$N$ & 3.37 & 3.34 & 1.24 & 5.70 & 0.96 \\
\hline$A R$ & 0.88 & 0.76 & 0.31 & 3.70 & 0.46 \\
\hline BN & 1.11 & 1.05 & 0.90 & 1.87 & 0.17 \\
\hline$H W$ & 0.77 & 0.72 & 0.30 & 1.99 & 0.28 \\
\hline
\end{tabular}

$V$-Volume, $\mathrm{H}$ - height, $\mathrm{W}$ - mean, maximal diameter of the dome perpendicular to $\mathrm{H}, \mathrm{N}$ - mean diameter of the neck, $A R$ - aspect ratio, $B N-$ bottle neck ratio, HW - height to width ratio.

Table IV. Aneurysm locations presenting highest dome size parameters

\begin{tabular}{|lccccccccc|}
\hline \multirow{2}{*}{ Variable } & \multicolumn{2}{c}{ Volume } & \multicolumn{2}{c}{ Height } & \multicolumn{2}{c}{ Dome width } & \multicolumn{2}{c|}{ Neck } \\
\cline { 2 - 11 } & Average & Std. dev. & Average & Std. dev. & Average & Std. dev. & Average & Std. dev. \\
\hline ICA cave (C5) & 52.57 & 20.03 & 3.632 & 0.773 & 4.536 & 0.546 & 3.874 & 0.190 \\
\hline ICA ophth. (C6) & 46.09 & 14.95 & 3.873 & 0.318 & 4.530 & 0.726 & 4.243 & 0.740 \\
\hline BA tip & 35.39 & 9.52 & 2.655 & 0.292 & 4.818 & 0.567 & 4.639 & 0.614 \\
\hline ICA p. com. (C7) & 30.55 & 5.60 & 2.995 & 0.283 & 4.144 & 0.263 & 3.865 & 0.269 \\
\hline
\end{tabular}

Volume in $\mathrm{mm}^{3}$, other parameters in $\mathrm{mm}$. Ophth. - ophthalmic, p. com. - posterior communicating.

Table V. Aneurysm locations with highest shape coefficients

\begin{tabular}{|lcccccc|}
\hline Variable & \multicolumn{3}{c}{ AR } & \multicolumn{2}{c|}{ BN } & HW \\
\cline { 2 - 7 } & Average & Std. dev. & Average & Std. dev. & Average & Std. dev. \\
\hline ACA (PeCA) & 2.110 & 0.799 & 1.436 & 0.223 & 1.378 & 0.303 \\
\hline ACA (A1) & 1.912 & 0.035 & 1.334 & 0.110 & 1.445 & 0.145 \\
\hline ICA (bif.) & 1.367 & 0.242 & 1.183 & 0.087 & 1.141 & 0.164 \\
\hline ALL & 0.878 & 0.457 & 1.109 & 0.165 & 0.770 & 0.280 \\
\hline
\end{tabular}

PeCA - Pericallosal artery, bif. - bifurcation. 
municating (C7) aneurysms presented the highest average values of all dome size parameters (Table IV).

Analysis of shape coefficients showed that ACA aneurysms in both proximal (A1) and distal (PeCA) locations and also ICA bifurcation aneurysms present the highest values of AR, BN and HW (Table V).

\section{General analysis of dome size changes}

We found one aneurysm located on an ICA bifurcation which thrombosed completely. It was assigned to the group of aneurysms reducing their size but excluded from calculations concerning speed of dome size parameters' change in order to avoid underestimation of these parameters.

Twenty-three (20.9\%) aneurysms significantly increased their volume. Ten (9.1\%) aneurysms presented a significant increase of the $H$ parameter and 14 $(12.7 \%)$ a significant increase of the $W$ dimension.

We compared the detection of dome growth between each assessment. The results are summarized in Table VI.

We observed that in comparison to other types of assessment, volume change analysis allows one to detect the highest percentage of significant aneurysm dome size increase in other types of assessment.

Variance analysis (ANOVA) between groups of aneurysms according to their size parameters' change, for each parameter separately did not reveal

Table VI. Detection of significant increase between all used methods

\begin{tabular}{|lccc|}
\hline $\begin{array}{l}\text { Group of significant } \\
\text { increase in }\end{array}$ & \multicolumn{3}{c|}{$\begin{array}{l}\text { Probability of detection by use of } \\
\text { other methods of assessment [\%] }\end{array}$} \\
\cline { 2 - 4 } & V & $H$ & $W$ \\
\hline$V(n=23)$ & - & 39.13 & 56.52 \\
\hline$H(n=10)$ & 90 & - & 40 \\
\hline$W(n=14)$ & 92.86 & 28.57 & - \\
\hline$H$ and $W(n=20)$ & 90 & 50 & 70 \\
\hline $\begin{array}{l}V-\text { Volume assessment, } \\
\text { diameter assessment. }\end{array}$
\end{tabular}

any significant differences in initial size or shape of aneurysms. Also multivariate regression analysis including accepted risk factors and dome size and shape initial parameters did not build any trusted model which could predict dome growth.

Annual risk of aneurysm volume increase in the presented group was estimated for 6.9\%/year (23 cases during 333.32 total aneurysm-years of observation).

\section{Analysis of dome size change for specific locations}

The percentage of growing aneurysms varies between anterior and posterior circulation (Table VII). None of the presented differences was statistically significant $\left(\chi^{2}>0.4\right)$.

All parameters describing speed of dome size change were significantly higher for posterior circulation aneurysms ( $p<0.0037$, Table VIII).

The basilar tip aneurysms present the highest coefficients of speed of dome size change compared to other locations. Average $\mathrm{dV} / \mathrm{d} T$ for these aneurysms was $0.3355 \mathrm{~mm}^{3} /$ day.

\section{Time to significant dome size change analysis}

Assuming constant speed of dome size change and Cls for a particular aneurysm, we calculated the expected time to dome size change for each grow-

Table VII. Differences in percentage of growing aneurysms between anterior and posterior circulation for each analysis

\begin{tabular}{|lccc|}
\hline Variable & \multicolumn{2}{c|}{ Size increase [\%] } & $\chi^{2}$ \\
\cline { 2 - 3 } & Anterior & Posterior & \\
\hline V analysis & 20 & 30 & 0.46392 \\
\hline H analysis & 8 & 20 & 0.40182 \\
\hline W analysis & 12 & 20 & 0.64580 \\
\hline
\end{tabular}

Table VIII. Difference in dome growth speed parameters between anterior and posterior circulation

\begin{tabular}{|lccccccc|}
\hline \multirow{2}{*}{ Parameter } & \multicolumn{2}{c}{ Average } & \multicolumn{2}{c}{ Number } & \multicolumn{2}{c}{ Std. dev. } & \multirow{2}{*}{ Value of $p$} \\
\cline { 2 - 6 } & Anterior & Posterior & Anterior & Posterior & Anterior & Posterior & \\
\hline $\mathrm{d} V / \mathrm{d} T$ & 0.001675 & 0.167982 & 99 & 10 & 0.02892 & 0.48624 & 0.000708 \\
\hline $\mathrm{d} H / \mathrm{d} T$ & -0.000046 & 0.001950 & 99 & 10 & 0.00108 & 0.00528 & 0.001535 \\
\hline $\mathrm{d} W / \mathrm{d} T$ & 0.000052 & 0.001384 & 99 & 10 & 0.00111 & 0.00289 & 0.003668 \\
\hline
\end{tabular}


ing aneurysm, separately for $V, H$ and $W$ assessment using equation 8.

The analysis was performed using Kaplan-Meier graphs. First, we analyzed three significantly increasing size groups of aneurysms $(\mathrm{V}, \mathrm{H}$ and $\mathrm{W}$ assessment separately). Twentieth, $50^{\text {th }}$ and $75^{\text {th }}$ percentile points for each parameter were calculated. The results are presented in Table IX and show that volume analysis has the shortest time to detection up to the $50^{\text {th }}$ percentile. For the $75^{\text {th }}$ percentile $H$ analysis shows almost similar time as $V$.

The same calculations were repeated for $H$ and $W$ assessment in the $V$ increasing group. Results are presented in Table $X$.

These calculations revealed that relying only on the $H$ analysis could delay detection of the same number of growing aneurysms more than four-fold for each percentile. The $W$ analysis performs better, but also could cause a two-to-three-fold delay.

A comparison of time of detection of aneurysm growth using Kaplan-Meier graphs between the an- terior and posterior circulation was not possible due to a low $(n=3)$ number of growing aneurysms in the posterior circulation. This analysis was performed using average values of eT and $t$-tests. Results are presented in Table XI.

The results, especially for $V$ assessment, were quite surprising for our team, considering the significant differences in speed of dome size changes. This paradox was solved in the analysis of confidence intervals, which shows accuracy of measurements for these groups separately. For each analyzed parameter these intervals were higher in the posterior circulation. For $V$ analysis the factor of difference was close to 10.0 (!) and for $H$ and $W$ above 2.0.

\section{Aneurysm bleeding during the time of observation}

During the period of follow-up bleeding of three aneurysms was noted (ACOA, BA, ICA at PCOA). It comprised $2.7 \%$ of all observed aneurysms $(n=110)$. Within the cumulative follow-up period of 333.32

Table IX. $25^{\text {th }}, 50^{\text {th }}$ and $75^{\text {th }}$ percentile points of detection of significant dome size change for $V, H$ and $W$ assessment. Growth detection specific for analyzed dome size parameter

\begin{tabular}{|c|c|c|c|c|c|c|}
\hline \multirow[t]{3}{*}{ Assessment } & \multicolumn{6}{|c|}{ Percentile } \\
\hline & \multicolumn{2}{|c|}{$25^{\text {th }}$} & \multicolumn{2}{|c|}{$50^{\text {th }}$} & \multicolumn{2}{|c|}{$75^{\text {th }}$} \\
\hline & Days & Years & Days & Years & Days & Years \\
\hline V & 213.85 & 0.59 & 711.21 & 1.95 & 1071.20 & 2.93 \\
\hline$H$ & 628.60 & 1.72 & 820.58 & 2.25 & 976.26 & 2.67 \\
\hline W & 324.39 & 0.89 & 724.97 & 1.99 & 1277.42 & 3.50 \\
\hline
\end{tabular}

Table X. $25^{\text {th }}, 50^{\text {th }}$ and $75^{\text {th }}$ percentile points of detection of significant dome size change for $H$ and $W$ assessment in increasing volume of dome group of aneurysms

\begin{tabular}{|c|c|c|c|c|c|c|}
\hline \multirow[t]{3}{*}{ Assessment } & \multicolumn{6}{|c|}{ Percentile } \\
\hline & \multicolumn{2}{|c|}{$25^{\text {th }}$} & \multicolumn{2}{|c|}{$50^{\text {th }}$} & \multicolumn{2}{|c|}{$75^{\text {th }}$} \\
\hline & Days & Years & Days & Years & Days & Years \\
\hline$H$ & 975.37 & 2.67 & 1497.81 & 4.10 & 3121.33 & 8.55 \\
\hline W & 418.95 & 1.15 & 1010.40 & 2.77 & 3907.17 & 10.70 \\
\hline
\end{tabular}

Table XI. T-test results for comparison of anterior and posterior circulation aneurysms' expected time to significant dome size change for each type of assessment

\begin{tabular}{|c|c|c|c|c|c|c|c|}
\hline \multirow[t]{2}{*}{ Assessment } & \multicolumn{2}{|c|}{ Average eT [days] } & \multirow[t]{2}{*}{ Value of $p$} & \multicolumn{2}{|c|}{ Number } & \multicolumn{2}{|c|}{ Standard deviation } \\
\hline & Ant. c. & Post. c. & & Ant. c. & Post. c. & Ant. c. & Post. c. \\
\hline V & 673 & 801 & 0.724 & 20 & 3 & 488.9 & 1108.2 \\
\hline$H$ & 2465 & 1269 & 0.420 & 20 & 3 & 2400.1 & 1818.2 \\
\hline W & 2442 & 2699 & 0.910 & 20 & 3 & 3574.9 & 4130.2 \\
\hline
\end{tabular}


aneurysm-years the risk of bleeding was estimated at $0.9 \%$ per year per aneurysm.

In the ruptured group all aneurysms presented a significant increase of volume. Two of the ruptured aneurysms developed daughter sacs (ACoA and BA) visible in post-bleeding studies. No daughter sac formation or its presence was observed in the non-bleeding group.

The initial size parameters of ruptured aneurysms were slightly greater than the rest of the volume increasing group $(n=32)$, but these differences were not statistically significant. Dome shape parameters were similar for both groups (Table XII).

Bleeding aneurysms grew significantly faster than the unruptured group ( $p \leq 0.000065)$. Changes of $A R, B N$ and HW coefficients and also their speed were higher in the bleeding group, and the differences were statistically significant for all parameters
( $p<0.46$ ), except $\mathrm{d} H W / \mathrm{d} T$ (Table XIII). Multivariate regression analysis of risk factors, aneurysm size and shape parameters and also their change did not produce a trusted model and did not reveal any other risk factors for aneurysm rupture.

For two of the three bleeding aneurysms there were intermediate CTA studies: one for ACOA aneurysm and three for ICA PCOA aneurysm.

Analysis of the obtained data showed that for ACoA aneurysm a significant and fast increase in volume was observed between all available studies. The speed of volume change was $0.08 \mathrm{~mm}^{3} /$ day between the first and the second study and $0.31 \mathrm{~mm}^{3}$ / day between the second and the third study.

For ICA-PCOA aneurysm we observed around $0.01 \mathrm{~mm}^{3} /$ day increase in volume between the first and second study ( $d T=263$ days). After that time the volume remained stable for more than 3.5 years.

Table XII. Characteristics of initial size and shape of bleeding and non-bleeding groups of aneurysms

\begin{tabular}{|c|c|c|c|c|c|}
\hline \multirow[t]{2}{*}{ Parameter } & \multicolumn{2}{|c|}{ Average } & \multicolumn{2}{|c|}{ Std. dev. } & \multirow[t]{2}{*}{ Value of $p$} \\
\hline & Non-bleeding & Bleeding & Non-bleeding & Bleeding & \\
\hline V & 20.00 & 38.76 & 18.233 & 6.069 & 0.089121 \\
\hline$H$ & 2.63 & 3.40 & 1.018 & 0.313 & 0.205482 \\
\hline W & 3.42 & 4.64 & 1.157 & 0.444 & 0.081788 \\
\hline$N$ & 3.14 & 4.36 & 1.227 & 0.684 & 0.103792 \\
\hline$A R$ & 0.974 & 0.794 & 0.685 & 0.134 & 0.656392 \\
\hline$B N$ & 1.127 & 1.076 & 0.192 & 0.107 & 0.650176 \\
\hline$H W$ & 0.816 & 0.739 & 0.377 & 0.118 & 0.729493 \\
\hline
\end{tabular}

Table XIII. Characteristics of size and shape change of bleeding and non-bleeding groups of aneurysms

\begin{tabular}{|lccccc|}
\hline Parameter & \multicolumn{2}{c}{ Average } & \multicolumn{2}{c}{ Std. dev. } & \multirow{2}{*}{ Value of $p$} \\
\cline { 2 - 5 } & Non-bleeding & Bleeding & Non-bleeding & Bleeding \\
\hline $\mathrm{d} V / \mathrm{d} T$ & 0.01408 & 0.58673 & 0.02122 & 0.838931 & 0.000065 \\
\hline $\mathrm{d} H / \mathrm{d} T$ & 0.00041 & 0.00731 & 0.00042 & 0.008606 & 0.000008 \\
\hline $\mathrm{d} W / \mathrm{d} T$ & 0.00069 & 0.00445 & 0.00082 & 0.004414 & 0.000058 \\
\hline $\mathrm{d} A R$ & 0.01007 & 0.60591 & 0.25220 & 0.774894 & 0.003176 \\
\hline $\mathrm{d} B N$ & 0.01954 & 0.28294 & 0.10837 & 0.288792 & 0.001598 \\
\hline $\mathrm{d} H W$ & 0.01103 & 0.25975 & 0.15418 & 0.387676 & 0.026484 \\
\hline $\mathrm{d} A R / \mathrm{d} T$ & -0.00017 & 0.00092 & 0.00086 & 0.000978 & 0.045641 \\
\hline $\mathrm{d} B N / \mathrm{d} T$ & -0.00003 & 0.00045 & 0.00022 & 0.000401 & 0.002135 \\
\hline $\mathrm{d} H W / \mathrm{d} T$ & -0.00009 & 0.00039 & 0.00042 & 0.000483 & 0.074283 \\
\hline
\end{tabular}


Two years after that time and more than 4 years after the start of observation, between the last follow-up study and CTA performed after bleeding a significant increase in average speed of growth was observed up to $0.04 \mathrm{~mm}^{3} /$ day.

The analysis of maximum values of $\mathrm{d} V / \mathrm{d} T, \mathrm{~d} H /$ $\mathrm{d} T$ and $\mathrm{d} W / \mathrm{d} T$ between the bleeding and non-bleeding groups of aneurysms showed that none of the non-bleeding aneurysms grew at a faster speed than $0.1 \mathrm{~mm}^{3} /$ day in volume, $0.002 \mathrm{~mm} /$ day in $H$ and $0.0034 \mathrm{~mm} /$ day in $W$. When analyzing the intermediate studies, all bleeding aneurysms at least in one pair of compared studies exceeded these limits.

\section{Discussion}

This is a single-center, retrospective study, which is a disadvantage.

The methods for assessment of significant aneurysm dome size changes proposed by our team are unique compared to previous studies concerning the problem of aneurysm growth. We decided to use this method because the accuracy of measurements of the dome of the aneurysm in any imaging study is a derivative of two independent accuracies. The first is the accuracy of the CTA study. It consists of scanner spatial resolution, type of reconstruction kernel, time of scanning (aneurysm size and orientation change during cardiac cycle) and also concentration and spatial distribution of contrast in the assessed vessels. The second is accuracy of the act of measurement, which depends on the used tools, abilities and experience of the person who performs it and also the specific configuration of the aneurysm and surrounding vessels.

Relying only on CTA study accuracy, for example in the present series, could lead to overestimation of significant dome size change for posterior circulation aneurysms. On the other hand, when the rule of Cls was the only one applied, the proportion of aneurysms changing size, especially reducing it, was very high, reaching $20 \%$ in $\mathrm{V}$ assessment. But when the $\mathrm{Cls}$ for these aneurysms were analyzed, we found that most of them had Cls close to $0.1 \mathrm{~mm}$. This level of accuracy could be real for used measuring tools but definitely not for current CT scanners.

Our measuring method using two dedicated tools for dome and neck measurements performed very well; the average accuracy of measurement was close to $0.4 \mathrm{~mm}$ and for most of them it was below
$0.625 \mathrm{~mm}$. The main limitation of measurements was the accuracy of the CT scanner. On the other hand, it is worth mentioning that in 10 cases this accuracy was above $1 \mathrm{~mm}$ and for 2 above $3 \mathrm{~mm}$. Almost all of these aneurysms had a poorly defined neck (ACOA, MCA bifurcation and BA bifurcation) or were situated close to the bone of the sella turcica or clivus.

These examples show the importance of assessment of the accuracy of measurements, especially in the case of difficult morphology of the dome and neck.

The annual risk of growth calculated for our series (6.9\% per year), taking into account the analyzed subset of unruptured aneurysms $(<7 \mathrm{~mm})$, is similar to the previous studies $[13-18,21,22]$ in which the annual risk of growth for these aneurysms was estimated at between 2.55\% [14] and 8.04\% [21].

The presented results provide evidence that the volume change assessment presents the highest percentage (>90\%) of detection of significant dome size change in $H$ and $W$ separately and also combined with the logical AND operator.

Despite the high percentage of patients with arterial hypertension, smoking and previous SAH history, we did not confirm that these factors increase the risk of aneurysm growth or rupture. Other factors also did not present a significant influence on any of the risks.

The influence of AR and other investigated dome shape coefficients on aneurysm growth and rupture, found in previous studies [12,13], is not evaluable in the presented material. This is due to the fact that most of the observed aneurysms in this study presented all coefficients very close to or below 1.0.

Our results confirmed different speed of dome size change between anterior and posterior circulation aneurysms $[15,18]$. According to the assessment of accuracy of measurements, we found that despite these differences detection of significant growth in the posterior circulation does not happen significantly faster than in the anterior circulation.

Risk of bleeding estimated at $0.9 \%$ per year per aneurysm is in accordance with previously reported values [3]. The group of ruptured aneurysms presented significantly higher speed of dome growth than other growing aneurysms. This confirms previous results [23]. Significant differences in dome shape coefficients' change between both groups were also found. The fastest changing coefficient was AR. Transforming these data into geometry, bleeding 
aneurysms seems to elongate before rupture. This process could be partially due to daughter sac formation. Two of three bleeding aneurysms presented this feature. This observation is in accordance with previous results mentioning daughter sac formation among the most important risk factors of bleeding $[13,16,24]$. Unfortunately in the present series the formation of daughter sacs was visible only in post-bleeding studies so prevention of bleeding using this feature was not possible.

The detailed analysis of dome size change in consecutive studies for two of the bleeding aneurysms shows two different behaviors of the sac. In the first case we observed faster than average for growing group speed of volume increase. In the second case the sac showed growth between the first and second study. After that time a long period (about 3.5 years) of stability was observed. Finally we noticed a very fast increase in size, mostly due to daughter sac formation, in the last few months before rupture.

The presented results lead to the conclusion that assessment of speed of dome size parameters' change could be essential in prevention of rupture. These parameters should be evaluated each time between consecutive follow-up studies.

The next conclusion is that ascertainment of dome size stability for even a long period of time could not be a justification for prolongation of intervals between imaging studies.

The proper interval between imaging studies still cannot be estimated based on the presented results. What we have found is that $50 \%$ of increasing volume aneurysms could be identified in less than two and $75 \%$ in less than 3 years of observation. Estimation of the optimal interval between studies will require further analyses, at least assessment of the dome size and shape in all available studies.

\section{Conclusions}

Dome volume assessment proved to be superior in aneurysm size change detection compared to only height and width assessment.

For best possible accuracy, the size of the aneurysm dome and neck should be calculated from multiple measurements, optimally using dedicated tools. A growth detection threshold algorithm has to take into account not only the CT scanner spatial resolution but also accuracy of measurements (Cls), especially for posterior circulation aneurysms.
The two most important predictors of rupture are daughter sac formation and fast increase in size estimated for the analyzed population of aneurysms for: $0.1 \mathrm{~mm}^{3} /$ day, $0.002 \mathrm{~mm} /$ day and $0.0034 \mathrm{~mm} /$ day respectively for $V, H$ and $W$ assessment. Speed of each dome size parameter change should be evaluated for each control.

Detection of daughter sac formation is a strong predictor of rupture and should lead to aneurysm treatment. In the first 3 years of observation we expect to detect up to $75 \%$ of volume increasing aneurysms.

Even a long period of dome stability is not justification for prolongation of the interval between follow-up studies or stopping observation.

\section{Conflict of interest}

The authors declare no conflict of interest.

\section{References}

1. International Study of Unruptured Intracranial Aneurysms Investigators. Unruptured intracranial aneurysms - risk of rupture and risks of surgical intervention. N Engl J Med 1998; 339: 1725-33.

2. Wiebers DO, Whisnant JP, Huston J III, et al.; International Study of Unruptured Intracranial Aneurysms Investigators. Unruptured intracranial aneurysms: natural history, clinical outcome, and risks of surgical and endovascular treatment. Lancet 2003; 362: 103-10.

3. Morita A, Kirino T, Hashi K, et al. The natural course of unruptured cerebral aneurysms in a Japanese cohort. N Engl J Med 2012; 366: 2474-82.

4. Rinkel GJ, Algra A. Long-term outcomes of patients with aneurysmal subarachnoid haemorrhage. Lancet Neurol 2011; 10 : 349-56.

5. Brown RD. Unruptured intracranial aneurysms. Semin Neurol 2010; 30: 537-44.

6. McDonald JS, McDonald RJ, Fan J, et al. Comparative effectiveness of unruptured cerebral aneurysm therapies: propensity score analysis of clipping versus coiling. Stroke 2013; 44: 988-94.

7. Greving JP, Wermer MJ, Brown RD Jr, et al. Development of the PHASES score for prediction of risk of rupture of intracranial aneurysms: a pooled analysis of six prospective cohort studies. Lancet Neurol 2014; 13:59-66.

8. Obara A, Dzikiewicz MA, Maruszyński M, et al. Lymphatic complications after vascular interventions. Videosurgery Miniinv 2014; 9: 420-6.

9. Poncyljusz W, Sagan L, Safranow K, Rać M. Initial experience with implantation of novel dual layer flow-diverter device FRED. Videosurgery Miniinv 2013; 8: 258-64.

10. Mocco J, Komotar RJ, Lavine SD, et al. The natural history of unruptured intracranial aneurysms. Neurosurg Focus 2004; 17: E3.

11. Wermer MJ, van der Schaaf IC, Algra A, Rinkel GJ. Risk of rupture of unruptured intracranial aneurysms in relation to pa- 
tient and aneurysm characteristics: an updated meta-analysis. Stroke 2007; 38: 1404-10.

12. Vlak MH, Algra A, Brandenburg R, Rinkel GJ. Prevalence of unruptured intracranial aneurysms, with emphasis on sex, age, comorbidity, country, and time period: a systematic review and meta-analysis. Lancet Neurol 2011; 10: 626-36.

13. Bor AS, Tiel Groenestege AT, terBrugge KG, et al. Clinical, radiological, and flow-related risk factors for growth of untreated, unruptured intracranial aneurysms. Stroke 2015; 46: 42-8.

14. Burns JD, Huston J 3rd, Layton KF, et al. Intracranial aneurysm enlargement on serial magnetic resonance angiography: frequency and risk factors. Stroke 2009; 40: 406-11.

15. Matsubara S, Hadeishi H, Suzuki A, et al. Incidence and risk factors for the growth of unruptured cerebral aneurysms: observation using serial computerized tomography angiography. J Neurosurg 2004; 101: 908-14.

16. Miyazawa N, Akiyama I, Yamagata Z. Risk factors for growth of unruptured intracranial aneurysms: follow-up study by serial 0.5-T magnetic resonance angiography. Neurosurgery 2006; 58: 1047-53.

17. Matsumoto K, Oshino S, Sasaki M, et al. Incidence of growth and rupture of unruptured intracranial aneurysms followed by serial MRA. Acta Neurochir (Wien) 2013; 155: 211-6.

18. Chien A, Liang F, Sayre J, et al. Enlargement of small, asymptomatic, unruptured intracranial aneurysms in patients with no history of subarachnoid hemorrhage: the different factors related to the growth of single and multiple aneurysms. J Neurosurg 2013; 119: 190-7.

19. Matsubara S, Hadeishi H, Suzuki A, et al. Incidence and risk factors for the growth of unruptured cerebral aneurysms: observation using serial computerized tomography angiography. J Neurosurg 2004; 101: 908-14.

20. Burns JD, Huston J III, Layton KF, et al. Intracranial aneurysm enlargement on serial magnetic resonance angiography: frequency and risk factors. Stroke 2009; 40: 406-11.

21. Villablanca JP, Duckwiler GR, Jahan R, et al. Natural history of asymptomatic unruptured cerebral aneurysms evaluated at CT angiography: growth and rupture incidence and correlation with epidemiologic risk factors. Radiology 2013; 269: 258-65.

22. Jeon JS, Ahn JH, Huh W, et al. A retrospective analysis on the natural history of incidental small paraclinoid unruptured aneurysm. J Neurol Neurosurg Psychiatry 2014; 85: 289-94.

23. Mehan WA Jr, Romero JM, Hirsch JA, et al. Unruptured intracranial aneurysms conservatively followed with serial CT angiography: could morphology and growth predict rupture? J Neurointerv Surg 2013 Nov 25. doi: 10.1136/neurintsurg-2013-010944.

24. Ujiie H, Tachibana $H$, Hiramatsu O, et al. Effects of size and shape (aspect ratio) on the hemodynamics of saccular aneurysms: possible index for surgical treatment of intracrania aneurysms. Neurosurgery 1999; 45: 119-29; discussion 129-30.

Received: 11.03.2015, accepted: 12.05.2015. 